\title{
A New Type of Gold Catalyst
}

\author{
ACTIVITY AND SELECTIVITY REGULATED \\ BY MEANS OF HOT ELECTRONS
}

\author{
Jan Figar and Walter Haidinger \\ Battelle Geneva Research Centre, Switzerland
}

Gold is generally considered to be a rather poor catalyst in heterogeneous reactions, this lack of activity usually being attributed to its chemical stability. For this reason, research into the properties of gold as an industrial catalyst has for long been neglected, but in the light of some recently published work by Professor Bond and his colleagues $(1,2)$, it seems to be worth while reconsidering the potential applications of gold, especially for the preparation of highly selective catalysts.

All metal surfaces might be supposed to have some catalytic activity, and as a rule of thumb the smaller the overall catalytic activity the higher the selectivity of the metal. This purely phenomenological statement is supported by some comparative studies of catalytic properties of the platinum group metals (3), while for gold it can be deduced from studies of gold-palladium alloys (2). In order to get a useful catalyst on the basis of gold, its "natural selectivity" (still speaking from the phenomenological point of view) must be preserved while its catalytic activity is enhanced. With this aim in mind, two useful guidelines for gold catalyst preparation can be deduced from the above-mentioned papers $(1,2)$, the first employing the effect of transmutation of catalytic properties of finely dispersed supported gold, the second making use of alloys or supported alloys in which the catalytic activity of a very active metal is tempered by gold. Unfortunately, inasmuch as the physical and chemical background of these two effects is still not fully understood, the research chemist is left with the trial-and-error method in looking for useful gold catalysts optimised in respect of a particular chemical reaction.

Recently another kind of catalytic behaviour of metal surfaces $(4,5)$, especially gold, has been investigated using a means to enhance catalytic activity which differs from the classical ones, i.e. a method of enhancing catalytic activity without destroying the high natural selectivity. The findings stemming from these investigations differ from other work essentially in the fact that an electrical current passes through the catalyst during the reaction.

As early as 1937, Lennard-Jones (6) supposed that conduction electrons could play a great part in surface reactions on metallic surfaces, namely in energytransferring processes, resulting in the activation of chemisorbed complexes. Increase of the energy carried by the electrons should manifest itself in an increase in the rate of reaction where the particular chemisorbed complexes take part. There is, however, no experimental foundation for this hypothesis, mainly for the reason that the total energy of conduction electrons is determined by the thermal energy, and field energies may be almost neglected, so that energy rise will always be accompanied by a temperature rise of the catalyst metal.

The basic idea for overcoming this difficulty is to produce an electric current which carries electrons with excess energy (energies above the Fermi level) by means other than temperature or field excitation. These electrons are called hot electrons, and can quite easily be produced by means of thin-layer devices well known in solid-state electronics. For experiments described in this paper a sandwich structure has been used, composed of a supporting metal base, covered with an insulating layer of alumina 50 to $1500 \AA$ thick, in turn covered with a top layer of gold (about $100 \AA$ thick) which is the catalytically active surface of the device. Only this top layer is in contact with the reactants.

When a small voltage is applied across such a device, the Fermi level is lowered on the positively biased top metal layer and electrons may pass through the insulator into the metal by direct tunnelling or by any other related effect. They will in fact lose energy during the passage through the metallic top layer towards the surface by electron-electron or electronphonon scattering processes, but when this top layer is not too thick a proportion of these electrons reach the surface with an energy greater than the Fermi energy. These hot electrons cannot persist in this excited state and must therefore drop back to the Fermi level, this being their only stable state of thermodynamic equilibrium. The excess energy of those electrons reaching the surface must be transferred to another particle, e.g. a chemisorbed complex, which is in turn activated to a higher energetic state. Hot electrons thus represent a source influencing the energy distribution in the surface population 
of chemisorbed complexes, providing an isothermal enhancement of the reaction rate. Although this mechanism is still very qualitative one might expect such devices to have two principal catalytic properties, namely that reaction-rate enhancement should be a function of the current density as well as of the excess energy of hot electrons, and that they should influence the selectivity of a reaction for systems where different kinds of chemisorbed complexes with different activation energies are involved.

The production of hot electrons over large areas by means of such devices is in reality a complex process, mainly for technological reasons, so that nothing can be deduced with precision about the energy distribution or about the fractional number of such hot electrons reaching the catalysing surface. However, the presence and the action of these electrons can be deduced directly from testing some chemical reactions with and without the flow of such electrons at otherwise almost constant conditions.

The metal of the top layer must obviously allow the chemisorption of at least one of the reactants taking part in the catalysed reaction. Nevertheless, as observed during these investigations, the natural catalytic activity of the top layer is of small importance; in fact, gold has been successfully used in our experiments in spite of its poor activity.

Two kinds of chemical reaction have been tested for this purpose, the decomposition of formic acid and the oxidation of propene. These were studied with and without hot electron flow through the catalyst. The experimental set-up, procedures and results are described in detail elsewhere $(4,5)$.

The formic acid decomposition was chosen to find an increase of activity, whereas the propene oxidation should cause activity enhancement as well as influencing the selectivity.

The rate of formic acid decomposition, measured by means of pressure increase in a static reactor, was increased in all experiments during electron flow. A typical experimental plot is shown in Figure 1. In some experiments the reaction rates obtained with electron flow were about 100 times higher than those without electron flow.

During these experiments, it has also been shown that these rate increases are related to the presence of hot electrons and are not due to conduction electrons. This was done by using devices without the inter- mediate insulation layer, so that the production of the hot electrons did not take place.

These results have thus shown that the hot electron flow has a considerable effect on the reaction rate of the catalysed reaction. A number of experiments were devoted to excluding any trivial sideeffect such as local heating of the catalytic device by heat dissipation. The results, summarised in (4) and (5), have shown that the rate increase cannot be explained by local heating.

In the oxidation of propene two different main reaction paths can occur, the first two stable intermediates being propylene oxide or acrolein. As reported, the heterogeneous reaction probably starts with the chemisorption of oxygen on gold $(7,8)$, and the propene is not chemisorbed on the proper surface of Group Ib metals. By analogy with silver (9), at least two distinct types of chemisorbed oxygen complexes exist simultaneously on gold surfaces, probably participating in different but parallel surface reactions. One or another of these complexes can thus be activated by hot electrons, the production of one or other type of stable reaction product thus being selectively accelerated. 


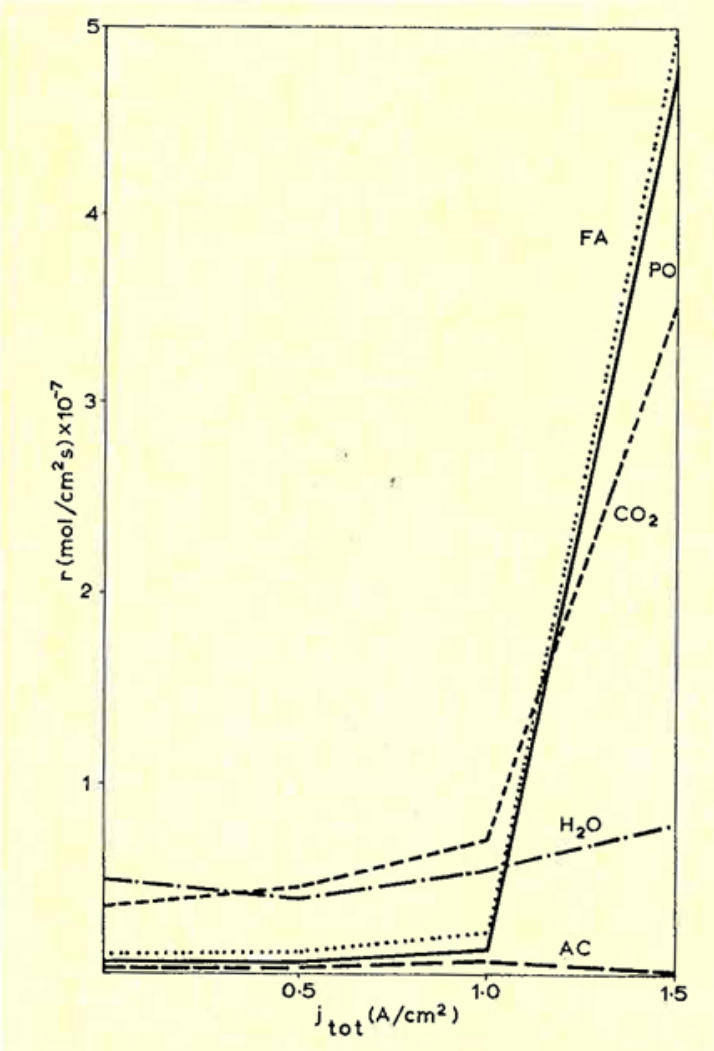

Fig. 2 Production-rate increase of principal oxidation products during propene oxidation as a function of the total current density.

PO propylene oxide; $A C$ acrolein; $F A$ formic acid

The reaction was carried out in a continuous reactor and checked by means of gas chromatography. Figure 2 shows the production rates in $\mathrm{mol} / \mathrm{cm}^{2} \mathrm{~s}$ of different reaction products as a function of current density passing through the catalytic device when a mixture of 73.6 volume per cent of propene and 26.4 volume per cent of oxygen was fed into the reactor at $315^{\circ} \mathrm{C}$.

From current density zero up to $1.0 \mathrm{~A} / \mathrm{cm}^{2}$ there is no significant variation of product composition, but above this value there is a marked enhancement of the overall oxidation rate of propene. At the same time, the production rates of different products are varied in a very different manner; the production rate of propylene oxide increased by a factor of 73.6, at the same time as that of $\mathrm{CO}_{2}$ increased by a factor of 10.4 and that of acrolein decreased to zero.

These results give strong evidence of what was expected on the basis of the hot electron mechanism. Taking them in connection with the results for the formic acid decomposition, and from the fact that such a poor catalyst as gold may be activated to such a high extent, we may conclude that there exists a nontrivial hot electron effect in heterogeneous catalysis by metal surfaces.
Although many problems remain unsolved with regard to these experiments, we feel that they open some very interesting prospects in heterogeneous catalysis, notably towards the development of very selective, "tunable" catalysts, working at relatively low temperatures, including both photocatalytic and biochemical types of reaction.

\section{References}

1 G. C. Bond and P. A. Sermon, Gold Bull, 1973, 6, 102-105

2 E. G. Allison and G. C. Bond, Catalysis Rev., 1972, 7, 233

3 P. N. Rylander and D. R. Steele, Engelhard Ind. Tech. Bull., 1960, 10, 17

4 W. Haidinger and J. Figar, Chem. Phys. Letters, 1971, 11, 545

$5 \mathrm{~J}$. Figar and W. Haidinger, Chem. Phys. Letters, 1973, 19, 564

6 J. E. Lennard-Jones and E. T. Goodwin, Proc. Roy Soc., 1937, A163, 101

7 V. E. Ostrovsky and N. N. Dobrovolsky, Preprints of Papers from IV Internat. Congress on Catalysis, Vol. 2, ed. J. W. Hightower (Houston, 1969), p. 823

8 P. C. Richardson and D. R. Rossington, F. Catalysis, $1971,20,420$

9 N. H. A. von Ham, B. E. Nieuwenhuys and W. M. Sachtler, F. Catalysis, 1971, 20, 408

\section{A Rhenium-Gold Petroleum Reforming Catalyst}

Some twenty-five years ago the introduction by Universal Oil Products of the first process for the catalytic reforming of low octane naphthas to motor fuel of high octane value led to a major change in the structure of the petroleum industry. A number of similar processes were soon developed, almost all of them employing a platinum catalyst on an alumina base to promote the several complex reactions involved, and reforming became firmly established as the basic method of providing the fuels needed by modern automobile engines. More recently the development of the "bimetallic" catalysts-the first of these relied upon the addition of rhenium to platinum-brought about a substantial improvement in stability, enabling longer runs to be achieved at higher space velocities with high yields.

Rhenium itself has been shown to possess appreciable activity as a reforming catalyst, but it has now been found that a rhenium-gold combination has a much greater activity than either of these metals alone. A recent disclosure by H. E. Merrill and R. S. Lunt of Esso Research and Engineering (U.S. Patent 3,785,960) claims a highly active reforming catalyst containing, for example, equal parts of rhenium and gold supported on an alumina base of high surface area. The octane rating of various hydrocarbon feedstocks can be successfully upgraded, according to the patent, at relatively low pressures to a value of above 99 in conventional reforming conditions. It is believed that the active catalyst is an alloy of rhenium and gold. 